\title{
Efecto de diferentes tratamientos superficiales en la alteración de color de la cerámica
}

\author{
Effect of different surface treatments on the alteration of color of ceramic
}

\author{
Jessica Bedoya Ocampoa ${ }^{\text {, }}$ Wilmer Bedoya Arias ${ }^{2 a}$, Carlos Andrés Solano Macías ${ }^{2 a}$, Andrea Bedoya \\ Ocampo $2 \mathrm{a}$
}

\section{RESUMEN}

Objetivo: El objetivo de este estudio fue evaluar el efecto de diferentes tratamientos de la superficie en la pigmentabilidad de la cerámica después de inmersión en solución de tinción. Materiales y Métodos: Se realizaron 44 discos de $10 \mathrm{~mm}$ de radio y $3 \mathrm{~mm}$ de espesura. Las muestras se asignaron aleatoriamente entre 4 grupos experimentales $(n=11)$ recibiendo tratamiento de la superficie de acuerdo a los diferentes grupos $M, H, B, L .(n=10)$ fueron distribuidas para evaluación del color y Rugosidad (RU) y una muestra de cada grupo utilizada para análisis por microscopia electrónica de barrido (MEB). Para (RU), los datos fueron sometidos a ANOVA on ranks $(\alpha=0.05)$, y para el color ANOVA un fator $(\alpha=0.05)$. En las microfotografías de la superficie se realizo análisis cualitativo/descriptivo. Resultados: En la (RU) comparada antes y después de la inmersión para cada grupo, no se observo diferencia estadística ( $p>0.05$ ). Al comparar los tipos de pulimento antes de la inmersión se observo diferencia entre el grupo $L$ con los demás $M, H, B(p<0.05)$. En el análisis de los grupos después de la inmersión, hubo una diferencia del grupo $L$ con $B$ y $M(p<0.05)$. Al comparar la alteración de color, después de la inmersión, hubo diferencia entre todos los grupos ANOVA $(\mathrm{p}<0.05)$. En el análisis por (MEB) todos los grupos presentaron superficies parcialmente porosas; sin embargo las barnizadas produjeron el mejor resultado grupos $\mathrm{H}$ y $\mathrm{B}$.

Conclusiones: La textura de la superficie es un factor importante en la pigmentabilidad de la cerámica. los materiales barnizados mostraron menor alteración del color.

Palabras claves: Cerámicas; Rugosidad; Color. (Fuente: DeCS BIREME)

\section{ABSTRACT}

Objective: The objective of this study was to evaluate the effect of different surface treatments on the pigmentability of the ceramic after immersion in staining solution. Materials and Methods: 44 disks of $10 \mathrm{~mm}$ radius and $3 \mathrm{~mm}$ thickness were made. Samples were randomly assigned among 4 experimental groups $(n=11)$ receiving surface treatment according to the different groups $M, H, B, L$. $(n=10)$ were distributed for color evaluation and Roughness $(R U)$ and a sample of each group used for scanning electron microscopy (SEM) analysis. For (RU), the data were subjected to ANOVA on ranks $(\alpha=0.05)$, and for the color ANOVA a factor $(\alpha=0.05)$. In the microphotographs of the surface, a qualitative/descriptive analysis was carried out. Results: In the (RU) compared before and after immersion for each group, no statistical difference was observed ( $p>$ $0.05)$. When comparing the types of polish before immersion, a difference was observed between the $L$ group with the other $M, H, B(p<0.05)$. In the analysis of the groups after immersion, there was a difference of group $L$ with $B$ and $M(p<0.05)$. When comparing the color alteration, after immersion, there was a difference between all the ANOVA groups $(p<0.05)$. In the analysis by (SEM) all groups had partially porous surfaces; however, the varnishes produced the best result groups $\mathrm{H}$ and $B$. Conclusions: The texture of the surface is an important factor in the pigmentability of ceramics. the varnished materials showed less color alteration.

Key words: Ceramics; Roughness; Color. (Source: MeSH NLM)

Aprobado: 20 de diciembre de 2018

Publicado: 30 de enero de 2019

${ }^{1}$ Escuela de Odontología, Doctorado, Universidad Estatal de Ponta Grossa, Ponta Grossa, PR, Brasil.

${ }^{2}$ Escuela de Odontología Universidad Santiago de Cali, Cali, Colombia.

a Odontólogo (a)

Correspondencia:

Jessica Bedoya

Correo electrónico: Jessica.bedoya.ocampo@gmail.com
Este es un artículo Open Access distribuido bajo la licencia Creative Commons Atribución-NoComercialCompartirlgual 4.0 


\section{INTRODUCCIÓN}

Las cerámicas son materiales ampliamente utilizados en la odontología restauradora debido a sus excelentes resultados estéticos, alta resistencia, biocompatibilidad y lisura superficial. Sin embargo, dependiendo del grado de lisura superficial, un aumento de la adherencia de bacterias puede ser visto sobre las superficies cerámicas y contribuir para el desarrollo de enfermedades en el ambiente oral. (1)

Se sabe que la acumulación de bacterias en áreas marginales de las cerámicas dentales pueden conducir a la formación de biofilm bacteriano, caries secundarias y a la inflamación periodontal subsecuente. $(2,3)$ Además, una textura superficial áspera o irregular reflejará un patrón de luz irregular y difuso, que modificará el color de la restauración,(4) lo que la hace más susceptible a manchas por pigmentos.(5)

Normalmente, antes del proceso de cementación, las restauraciones cerámicas requieren ajustes para eliminar interferencias oclusales, rectificación de los márgenes y contornos inadecuados para lograr una armonización funcional. $(6,7)$ Estos ajustes previos a la cementación, interrumpen la formación de una capa suave y lisa a través del proceso natural de glaseado además de eliminar la capa cristalina superficial, estos factores aumentan la rugosidad de la superficie, $(8,9)$ y pueden influir en el color de la cerámica. $(5,10-12)$

Se han realizado estudios sobre diferentes protocolos de pulido de las cerámicas (11-13), con el objetivo de definir el método de pulimento más adecuado para minimizar la rugosidad de la superficie y obtener una textura superficial lisa después de los ajustes. $(8,9)$ Algunos estudios indican que las superficies obtenidas a partir de los procedimientos de pulimiento y acabamiento, no son comparables con las superficies que reciben procedimiento de glaseado final. Otros, han demostrado que una secuencia de pulido puede producir una superficie igualmente lisa, e incluso estéticamente mejor. (13-15)

Estudios sobre la evaluación del color de las cerámicas (16) y la estabilidad del color (17) ya fueron desarrollados y se sabe que la estética y la estabilidad del color son factores importantes para el éxito de las restauraciones cerámicas(18). Por otro lado, se han realizado pocas investigaciones sobre los efectos del desgaste y el pulido sobre superficies de cerámicas sometidas a ajustes antes de su cementación y sobre la posible relación entre la alteración del color y la rugosidad de la superficie de la cerámica después de haber sido inmersa en solución de tinción $(17,19)$.

Por lo tanto, el objetivo de este estudio fue evaluar el efecto de diferentes tratamientos de la superficie, en la pigmentabilidad de la cerámica después de la inmersión en una solución de tinción.

\section{MATERIALES Y MÉTODOS}

Se realizaron 44 discos de $10 \mathrm{~mm}$ de radio y $3 \mathrm{~mm}$ de espesura de cerámica de recubrimiento sobre metal (IPS In line One Ivoclar Vivadent, Schaan, Liechtenstein), fueron fabricaron con ayuda de un conformador de muestra de cerámica (Smile line \# 7015, Saint-Imier, Suiza) de acuerdo con las instrucciones del fabricante. Posteriormente fueron retirados del conformador para ser llevadas a un horno Ceramat S (Jelrus, Búfalo, New Jersey) a una temperatura inicial de $450^{\circ} \mathrm{C}$ con una rata de ascenso de $55^{\circ} \mathrm{C}$ por minuto hasta $910^{\circ} \mathrm{C}$.

Después de la confección, todas las muestras fueron pulidas con piedra abrasiva troncocónica fina y gruesa de diamante sinterizado galvanizado (Jota Ag, Rotary Instrument, Suecia) por 15 segundos para estandarizar el tiempo de pulimento. Las muestras se asignaron aleatoriamente entre 4 grupos experimentales $(n=11)$ y se trataron de acuerdo a las diferentes condiciones de tratamiento de la superficie:

- Grupo (M): Adicionalmente a él pulimento con piedra abrasiva troncocónica fina y gruesa (Jota $\mathrm{Ag}$ ), que se realizó en todos los grupos, este grupo tuvo un glaseado manual con felpa de fieltro de lana natural impregnada con polvo abrasivo extrafino (Kuraray Noritake, Dental, Inc) y pasta de glase IPS Inline System (Ivoclare Vivadent, Schaan, Liechtenstein)

- Grupo (H): Fue pulido con disco de revestimiento de caucho impregnado con diamante multicapa (Exa Cerapol y Cerapol super, Edenta Ag, Suiza), adicional, este grupo recibió un glaseado manual con felpa de fieltro de lana natural impregnada con polvo abrasivo extrafino (Kuraray Noritake, Dental, Inc) y pasta de glase IPS Inline System (Ivoclare Vivadent, Schaan, Liechtenstein).

- Grupo (B): Un acabado con punta abrasiva ultra fina de diamante (Ceramic Technique System Kit, Shofu Inc) fue realizado en este grupo, además de un pulido con disco de revestimiento de caucho impregnado con diamante multicapa (Exa Cerapol y Cerapol Super, Edenta Ag, Suiza) y un glaseado manual con felpa de fieltro de lana natural impregnada con polvo abrasivo extrafino (Kuraray Noritake, Dental, Inc) y pasta de glase IPS Inline System (Ivoclare Vivadent, Schaan, Liechtenstein).

- Grupo (L): Fue pulido con disco de revestimiento de caucho impregnado con diamante multicapa (Exa Cerapol y Cerapol super, Edenta Ag, Suiza), y recibió un glaseado manual con felpa de fieltro de lana natural impregnada con polvo extrafino ( Kuraray Noritake, Dental, Inc) y pasta de piedra pómez compuesta por sílice y alúmina (Dentalia, Barranquilla, Colombia); 
además del glaseado manual, este grupo tuvo un glaseado adicional de acuerdo con las instrucciones del fabricante (IPS In line One Ivoclar Vivadent,
Schaan, Liechtenstein). Los grupos de pulimento están detallados en la tabla 1.

Tabla 1. Grupos de pulimento, elementos que los integran, composición, lote y fabricante

\begin{tabular}{|c|c|c|c|}
\hline Grupo & Elemento pulidor & Composición & Lote/Fabricante \\
\hline (M) & $\begin{array}{l}\text { Punta troncocónica } \\
\text { fina/gruesa } \\
\text { Felpa de fieltro } \\
\text { Pasta de glase IPS Inline } \\
\text { System }\end{array}$ & $\begin{array}{l}\text { Diamante sinterizado } \\
\text { galvanizado } \\
\text { Lana natural impregnada } \\
\text { con polvo abrasivo } \\
\text { extrafino } \\
\text { Glicerol }\end{array}$ & $\begin{array}{c}0567 / 8923 \text { (Jota Ag, Rotary } \\
\text { Instrument, Suecia) } \\
\text { 4545(Kuraray Noritake Dental Inc) } \\
0045 \text { (Ivoclare Vivadent, Schaan } \\
\text { Liechtenstein) }\end{array}$ \\
\hline$(\mathrm{H})$ & $\begin{array}{l}\text { Punta troncocónica fina/ } \\
\text { gruesa } \\
\text { Discos de revestimiento Exa } \\
\text { Cerapol/ Cerapol Super } \\
\text { Felpa de fieltro } \\
\text { Pasta de glase IPS Inline } \\
\text { System }\end{array}$ & $\begin{array}{l}\text { Diamante sinterizado } \\
\text { galvanizado } \\
\text { Caucho/Diamante } \\
\text { Multicapa } \\
\text { Lana natural impregnada } \\
\text { con polvo abrasivo } \\
\text { extrafino } \\
\text { Glicerol }\end{array}$ & $\begin{array}{c}\text { 0567/8923 (Jota Ag, Rotary } \\
\text { Instrument, Suecia) } \\
\text { 315/3489(Edenta Ag, Suiza) } \\
\text { 0287(Ivoclare Vivadent, Schaan, } \\
\text { Liechtenstein) }\end{array}$ \\
\hline (B) & $\begin{array}{l}\text { Punta troncocónica fina/ } \\
\text { gruesa } \\
\text { Punta abrasiva } \\
\text { Discos de revestimiento Exa } \\
\text { Cerapol/Cerapol Super } \\
\text { Felpa de fieltro } \\
\text { pasta de glase IPS Inline } \\
\text { System }\end{array}$ & $\begin{array}{c}\text { Diamante sinterizado } \\
\text { galvanizado } \\
\text { Diamante extrafino } \\
\text { Caucho /Diamante } \\
\text { Multicapa } \\
\text { Lana natural impregnada } \\
\text { con polvo abrasivo } \\
\text { extrafino } \\
\text { Glicerol }\end{array}$ & $\begin{array}{c}\text { 0567/8923 (Jota Ag, Rotary } \\
\text { Instrument, Suecia) } \\
\text { 14001 / (Ceramic Technique } \\
\text { system kit, Shofu Inc) } \\
\text { 315/489(Edenta Ag, Suiza) } \\
\text { 4545(Kuraray Noritake Dental Inc) } \\
\text { 0237 (Ivoclare Vivadent, Schaan, } \\
\text { Liechtenstein) }\end{array}$ \\
\hline$(\mathrm{L})$ & $\begin{array}{l}\text { Punta troncocónica fina/ } \\
\text { gruesa de revestimiento Exa } \\
\text { Discos de revol } \\
\text { Cerapol/Cerapol Super } \\
\text { Felpa de fieltro } \\
\text { Pasta de piedra pómez }\end{array}$ & $\begin{array}{l}\text { Diamante sinterizado } \\
\text { galvanizado (Jota Ag, } \\
\text { Rotary Instrument, } \\
\text { Suecia) } \\
\text { Caucho/Diamante } \\
\text { Multicapa } \\
\text { Lana natural impregnada } \\
\text { con polvo extrafino } \\
\text { Sílice y alúmina }\end{array}$ & $\begin{array}{c}\text { 0567/8923 (Jota Ag, Rotary } \\
\text { Instrument, Suecia) } \\
\text { 315/489(Edenta Ag, Suiza) } \\
\text { 4545/(Kuraray Noritake Dental } \\
\text { Inc) } \\
\text { 080/(Dentalia, Barranquilla, } \\
\text { Colombia) }\end{array}$ \\
\hline
\end{tabular}

Todos los elementos de pulido se usaron en una pieza de mano de baja rotación, girando a $15.000 \mathrm{rpm}$ durante 15 segundos y fueron realizados por un único operador previamente calibrado. Para evitar la reducción del espesor de los discos inferior a $3 \mathrm{~mm}$, durante el procedimiento de pulimento, todas las muestras se midieron frecuentemente con la ayuda de un calibrador digital de precisión (Absolute Digimatic Caliper; Mitutoyo, Kawasaki, Japón), y en seguida fueron lavadas con agua destilada en un limpiador ultrasónico (CD-4800 Digital Ultrasonic Cleaner, Jeken, Dongguan, China) durante 180 segundos.

Diez muestras fueron aleatoriamente seleccionadas para evaluar el color antes y después de la inmersión en la solución pigmentante, la prueba de Rugosidad (RU) también fue realizada antes y después de la inmersión en la solución pigmentante y una muestra de cada grupo fue utilizada para análisis de la superficie por microscopia electrónica de barrido (MEB), solo después de estar inmersas en la solución pigmentante.

Inmersión en la solución pigmentante bebida de café

Para las pruebas de Rugosidad y Color, las muestras fueron evaluadas antes y después de la inmersión en solución de café. 3,6 g de café instantáneo (Nescafe Tradicional, Bugalagrande, Colombia) fueron disueltos en $300 \mathrm{ml}$ de agua caliente e agitado cada 12 horas 
para evitar asentamiento. En seguida, los discos fueron inmersos consecutivamente por 12 días a $37^{\circ} \mathrm{C}$ para simular un año de consumo de café. $(20,21)$. Después de este periodo, las muestras de cerámica se lavaron con agua y se secaron con papel absorbente (Familia, Medellín, Colombia) y fueron evaluadas.

\section{Rugosidad (Ra)}

Fue utilizado un rugosímetro de contacto (modelo surftest-301, \# de serie 15700438, Mitutoyo- Japón), realizando lecturas en 5 regiones de la muestra, en una única superficie, tomándose como punto de referencia el centro de la muestra. Por último, fue obtenida la media aritmética de las 5 mediciones. La rugosidad fue realizada antes y después de la inmersión de las muestras en la solución de café.

Toma de Color / Preparación de la sustancia para Inmersión

Se tomaron los parámetros de color según el sistema CIEL * $a$ * $b$ * de la Comisión Internacional de Iluminación (l’Eclairage). Cada disco de cerámica fue medido con un espectrofotómetro (VITA Easyshadeß Compact, Bad Säckingen, Alemania) antes y después de la inmersión en solución de café. Después de la inmersión, las diferencias de color $(\Delta \mathrm{E})$ se calcularon con el espectrofotómetro (VITA Easyshade $\mathbb{B}$ Compact, Bad Säckingen, Alemania) con software (SpectraMagic 3.1; Minolta Co, Ltd, CyberChrome, Inc., Osaka, Japón), y se calculó el $\Delta \mathrm{E}$ de acuerdo con la fórmula $\Delta \mathrm{E} 2-1=([\Delta \mathrm{L}] 2+[\Delta \mathrm{a}] 2+[\Delta \mathrm{b}] 2) 1 / 2$.

\section{Microscopia Electrónica de Barredura (MEB)}

Para evaluar las alteraciones morfológicas que son producidas por los diferentes grupos de pulimento sobre las superficies de la cerámica, las microfotografías fueron realizadas en un solo momento, posterior a ser sometidas a los respectivos tratamientos de la superficie y a la inmersión en la solución de café, se tomó 1 disco de cada grupo y fueron desecados en sílice por 12 horas, revestidos con una aleación de oro-paladio y examinados en microscopía electrónica de barrido (MEB) por retro dispersión de electrones a 12 kV (VEGA 3 TESCAM, Shimadzu, Tóquio, Japón). Microfotografías de la superficie fueron realizadas a un aumento de 1000x para el análisis visual de las alteraciones superficiales de las muestras de cerámica.

\section{Análisis Estadístico}

El análisis estadístico fue realizado a través del programa Sigma Plot con un nivel de significancia de 0.05. Los datos fueron sometidos a la prueba de normalidad (Shapiro-Wilk). Para rugosidad, los datos obtenidos no superaron la prueba de normalidad, por eso se realizó el análisis estadístico con ANOVA on ranks. Para el color los datos si superaron la prueba de normalidad y se realizó el análisis estadístico con ANOVA un factor. Para las microfotografías de la superficie se realizó análisis cualitativo/descriptivo

\section{RESULTADOS}

Rugosidad ( $\mathrm{Ra})$

Tabla 2 - Valores medios de rugosidad (media y desviación estándar) de los grupos de pulimento evaluados antes y después de la inmersión en la bebida de café.

\begin{tabular}{c|c|c}
\hline Grupo & $\begin{array}{c}\text { Antes de Inmersión/ } \\
\text { bebida-café }\end{array}$ & $\begin{array}{c}\text { Después de la } \\
\text { inmersión/bebida-café }\end{array}$ \\
\hline $\mathrm{M}$ & $0.06 \pm 0.01 \mathrm{a} A$ & $0.07 \pm 0.04 \mathrm{a} \mathrm{B} \mathrm{A}$ \\
$\mathrm{H}$ & $0.07 \pm 0.01 \mathrm{a} \mathrm{A}$ & $0.07 \pm 0.01 \mathrm{a} \mathrm{B}$ \\
$\mathrm{B}$ & $0,07 \pm 0.01 \mathrm{a} \mathrm{A}$ & $0.06 \pm 0.01 \mathrm{a} \mathrm{B} \mathrm{A}$ \\
$\mathrm{L}$ & $0,17 \pm 0,03$ a B & $0.14 \pm 0.05 \mathrm{a} \mathrm{B}$ \\
\hline
\end{tabular}

\footnotetext{
*Análisis intra-grupo (antes y después de la inmersión) - letras minúsculas iguales en la misma línea significan ausencia de diferencia estadística; Análisis inter-grupo letras mayúsculas iguales en la misma línea significan ausencia de diferencia estadística

Para la prueba de rugosidad se realizó un primer análisis observando los momentos antes y después de la inmersión en la bebida para cada tipo de pulimento realizado. Los resultados indicaron que no se observó diferencia significativa $(p>0.05)$.

Al comparar los tipos de pulimento antes de la inmersión en la bebida, no se observó diferencia significativa entre los grupos $\mathrm{M}, \mathrm{H}, \mathrm{B}(\mathrm{p}>0.05)$, pero si se encontró diferencia entre el grupo $L$ con los demás $\mathrm{M}, \mathrm{H}, \mathrm{B}(\mathrm{p}<0.05)$.
} 
Para el análisis de los grupos de estudio después de la inmersión en la bebida, se encontró una diferencia significativa del grupo $L$ con grupo B y $M(p<0.05)$ y para los otros grupos, no se encontró diferencia significativa $(p>0.05)$.

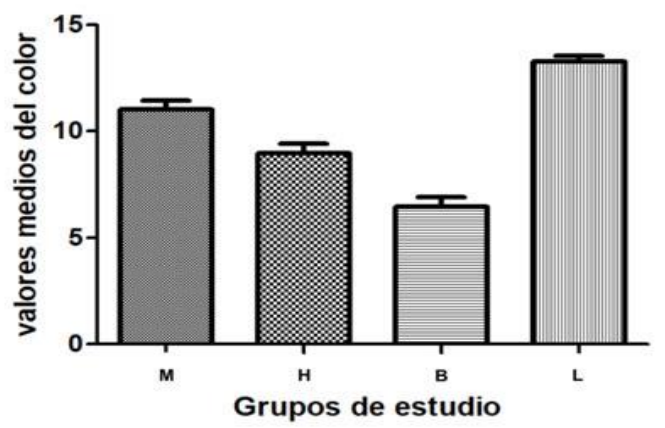

Figura 1- Medidas de variación del color $\Delta E$ y desviación estándar de los grupos de estudio.

En la prueba de color, la media y desviación estándar para los grupos de estudio fueron las siguientes: $\mathbf{M}$ $11.02 \pm 1.32$. H - $8.96 \pm 1.40$. B - $6.46 \pm 1.38$. L $13.27 \pm 0.85$.

Al comparar la alteración de color entre los grupos de estudio, después de la inmersión en la bebida, se observo diferencia estadística significativa entre todos los grupos ANOVA ( $\mathrm{p}<0.05)$.

\section{Microscopia Electrónica de Barredura (MEB)}

El análisis por microscopia electrónica de barredura mostro que todos los tratamientos dejaron las superficies parcialmente porosas y agrietadas; sin embargo las superficies barnizadas produjeron el mejor resultado grupos $\mathrm{H}$ y B figuras (2 y 3 ), el pulido con discos de revestimiento de caucho y diamante multicapa suavizo los cortes realizados con las puntas de diamante sinterizado galvanizado, sobre todo para el grupo B.

En los grupos $M$ y L figuras ( 1 y 4 ) el ajuste con el instrumento de corte de diamante creo las superficies mas ásperas alterando la topografía de la cerámica, generando relieves y surcos profundos. Las diferencias fueron significativas para el grupo $L$ que mostro la superficie más áspera, sucia, y con residuos de el material de pulimento empleado en este grupo para glasear (22) ( pasta de piedra pómez).

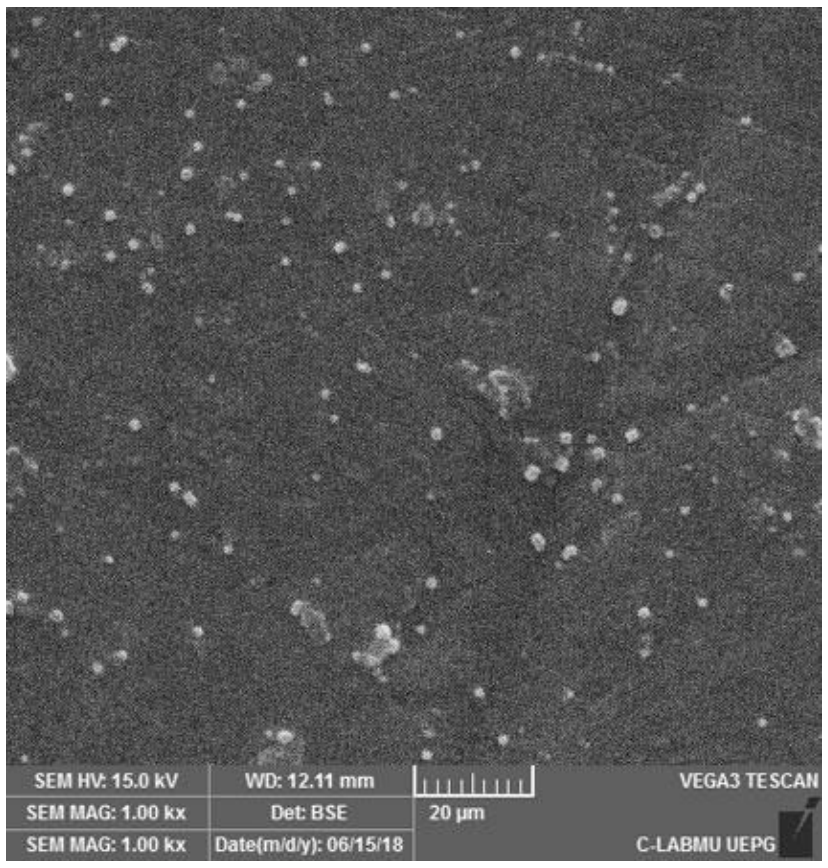

Figura 2. Fotomicrografía obtenida por MEB (Magnificación de 1000 veces) grupo M

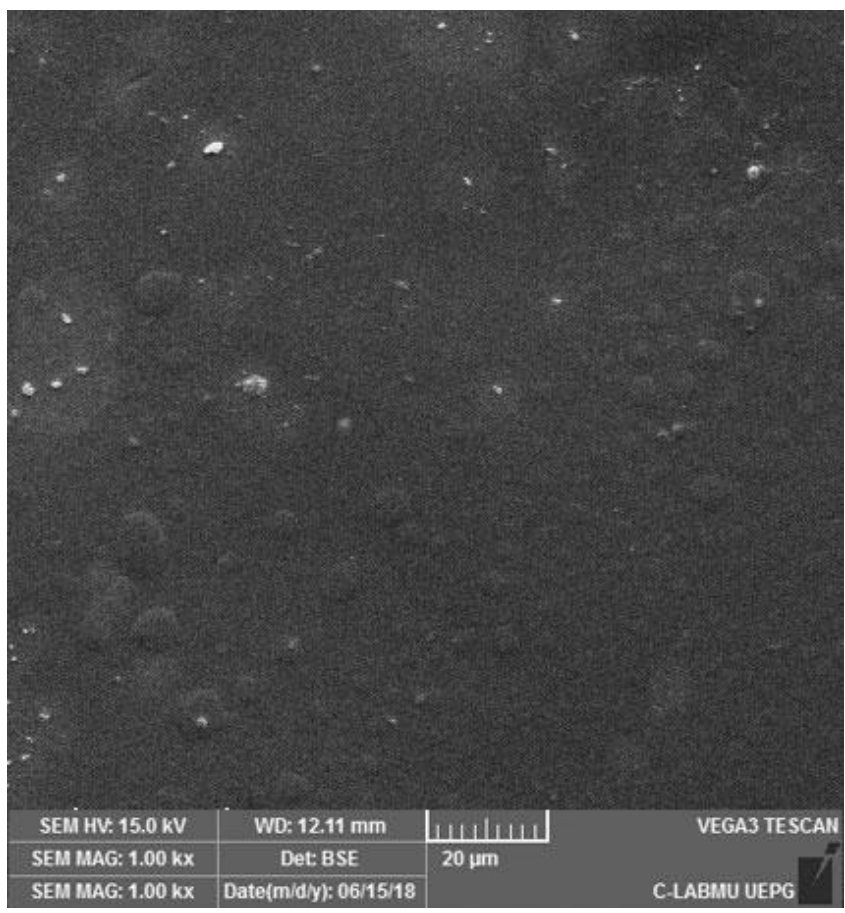

Figura 3. Fotomicrografía obtenida por MEB 

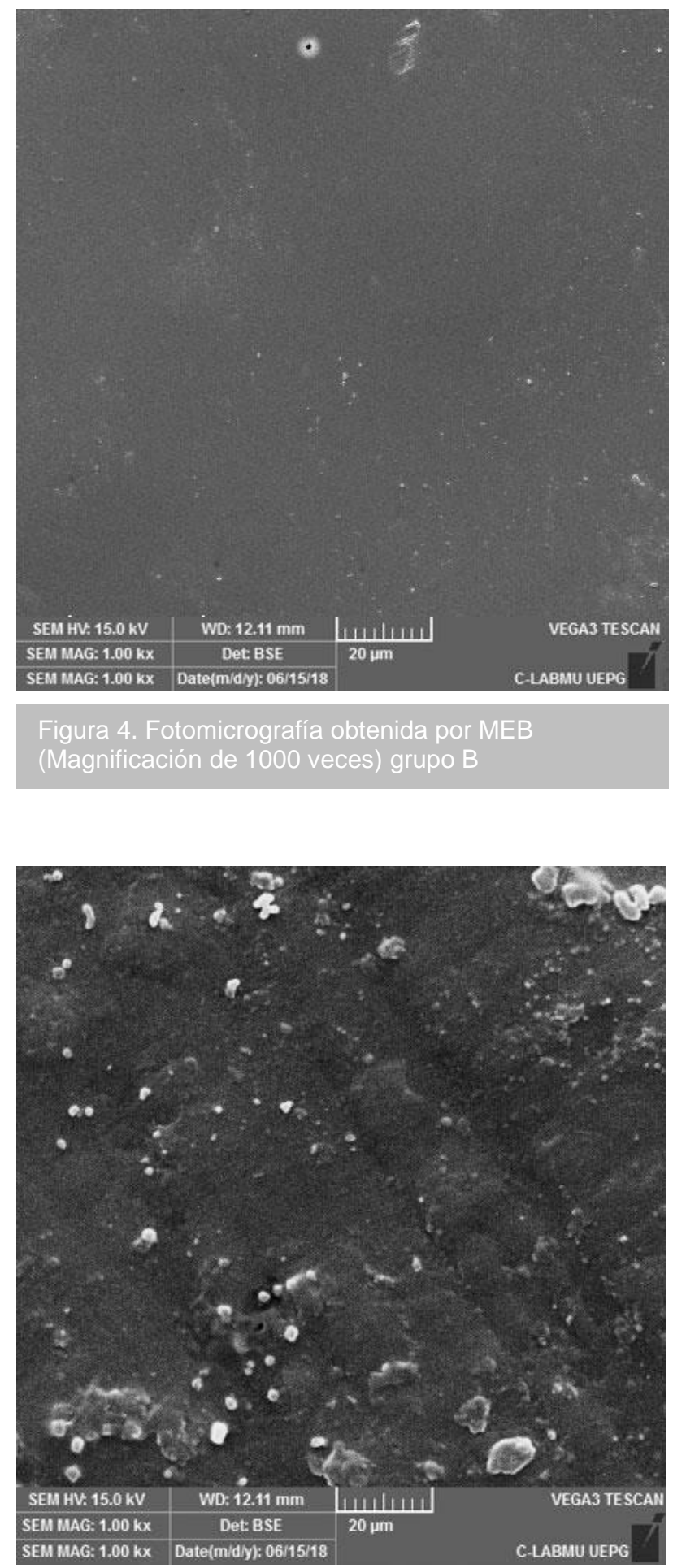

Figura 5. Fotomicrografía obtenida por MEB (Magnificación de 1000 veces) grupo L

\section{DISCUSIÓN}

Los grupos de estudio que mostraron una superficie más rugosa después del pulimento están directamente relacionados con la mayor alteración de color en este estudio.
Los ajustes realizados a una cerámica en momentos previos a su inserción crean una superficie rugosa, por esta razón investigadores han defendido la aplicaciónde un pulimento ideal para las restauraciones de cerámica, después de realizar el ajuste clínico (23-25). Estudios previos han mostrado que las técnicas y los sistemas de pulimento son incapaces de proporcionar superficies tan suaves como el esmalte $(26,27)$.

Fuzzi et al. (26) Demostró que superficies de cerámica glaseadas eran mas suaves, cuando comparadas con cerámicas que tuvieron otros sistemas de pulimento. Patterson et al. (28) Compararon efectos de sistemas de pulimento para el esmalte incluyendo la pasta diamantada, en este estudio la textura superficial reporto la rugosidad mas baja, estos hallazgos indican que los procedimientos de barnizado dieron como resultado una textura de superficie mas suave que los procedimientos de pulido/corte con puntas diamantadas. Se ha reportado desde hace un tiempo el uso de diferentes técnicas de pulido para restauraciones de cerámica después de realizar ajustes necesarios por factores estéticos o funcionales $(29,30)$. Otros autores (23) aconsejaron procedimiento de pulido de cuatro etapas, combinando pasta de diamante de grano fino y puntas de diamante de grano fino, como lo realizado en el presente estudio donde los hallazgos indicaron que el en el grupo de estudio B, el pulido llego a ser tan efectivo como el proceso de glaseado laboratorial.

Los Espectrofotómetros son los equipos actualmente utilizados para medir diferencias de color en los materiales dentales (31,32). Se eligió el Espectrofotómetro debido a su eficacia en la diferenciación del color sus resultados detallados y uso común. Esquivel et al. (22) Analizaron grupos de cerámicas glaseados y no glaseados sumergidos en solución de azul de metileno las evaluaciones colorimétricas que fueron compatibles con las visuales, indicaron que el grupo barnizado sufrió menos cambios de color que el grupo no barnizado.

Samra et al. (19) Afirmaron que después de la inmersión en café a $37^{\circ} \mathrm{C}$, durante 15 días la cerámica IPS Empress glaseada, mostro menos diferencia de color $(\Delta \mathrm{E}=1.28)$ comparada a las resinas compuestas. Otros estudios evaluaron los efectos del té, el café y la Coca-Cola en el color de resinas compuestas y cerámicas los autores encontraron que el color de la porcelana se modificó $(\Delta E=1.2$ a 1.4), pero no fue considerablemente perceptible $(33,34)$.

Los procedimientos de acabado y pulido pueden influir en el color de la cerámica $(5,11)$. Yilmaz et al. (5) Informaron que la cerámica pulida con discos de caucho de diamante junto con la pasta diamantada mostro más cambios de color que la porcelana glaseada sumergida en azul de metileno. En otro 
estudio, Atay et al. (33) Declararon que el tipo de tratamiento de superficie era un factor significativo en la estabilidad del color.

En muchos estudios, el té, el café y la Coca-Cola se han utilizado para experimentar pigmetabilidad en las cerámicas. $(20,33,34)$. En el presente estudio, el café se usó como sustancia de inmersión debido a su consumo frecuente en la vida diaria. (21) Según los autores La textura de la superficie afectó la coloración de la cerámica después de la inmersión en café. En el presente estudio, las superficies rugosas demostraron más cambios de color que las superficies lisas. todos los grupos mostraron una diferencia de color en un nivel que se consideraría clínicamente aceptable. La diferencia de color media del Grupo L, que tenía los valores más altos de rugosidad de la superficie, fue clínicamente inaceptable según el presente estudio. Debido a que las capas de esmalte se eliminaron después de la abrasión del instrumento de corte rotatorio con diamante, la superficie se volvió porosa y tendió a pigmentarse más, lo que afectó la estética de la cerámica.

En este estudio solo se usaron 1 agente de tinción y 1 tipo de cerámica. Como se ha relatado las diferentes soluciones afectan el color de la cerámica de manera diferente, la ausencia de varias soluciones de inmersión es una de las limitaciones del presente estudio. Diferentes resultados podrían haberse obtenido con diferentes tipos de cerámica. Es necesario realizar estudios adicionales utilizando varias soluciones de inmersión pigmentantes y diferentes tipos de cerámica.

Dentro de las limitaciones de este estudio in vitro, se puede concluir lo siguiente: La textura de la superficie es un factor significativamente importante en la pigmentabilidad de la cerámica. De acuerdo con los hallazgos, se puede concluir que los materiales glaseados y barnizados mostraron menor alteración del color. Sin embargo, el Grupo L (instrumento de corte rotatorio con diamante, más pasta de piedra pómez), fue clínicamente inaceptable. Se recomienda el barnizado o pulido de la superficie de la cerámica después de los ajustes que se realizan previos a la inserción definitiva.

Agradecimientos: A la Universidad Estatal de Ponta Grossa, por la contribución en el uso de equipos.

Contribuciones de autoría:

JBO, WBO, CASM: Participo en la concepción y diseño del estudio, obtención de datos, brindo asesoría estadística, redacción del artículo. ABO participo además en la revisión de literatura y análisis e interpretación de resultados. Todos los autores aprobaron a la versión final del manuscrito.

Fuente de financiamiento: financiamiento propio.
Conflictos de interés: Los autores no tienen ningun conflicto de interés en los resultados del estudio.

\section{REFERENCIAS}

1. Axelsson $\mathrm{P}$, Lindhe J. Effect of controlled oral hygiene procedures on caries and periodontal disease in adults. J Clin Periodontol. 1978;5(2):13351.

2. Meier R, Hauser-Gerspach I, Luthy $\mathrm{H}$, Meyer J. Adhesion of oral streptococci to all-ceramics dental restorative materials in vitro. Journal of materials science Materials in medicine. 2008;19(10):324953.

3. Rosentritt M, Behr M, Burgers R, Feilzer AJ, Hahnel S. In vitro adherence of oral streptococci to zirconia core and veneering glass-ceramics. Journal of biomedical materials research Part B, Applied biomaterials. 2009;91(1):257-63.

4. Lee YK, Lim BS, Kim CW. Effect of surface conditions on the color of dental resin composites. Journal of biomedical materials research. 2002;63(5):657-63.

5. Yilmaz C, Korkmaz T, Demirkoprulu H, Ergun G, Ozkan Y. Color stability of glazed and polished dental porcelains. Journal of prosthodontics : official journal of the American College of Prosthodontists. 2008;17(1):20-4.

6. Subasi MG, Alp G, Johnston WM, Yilmaz B. Effect of thickness on optical properties of monolithic CAD-CAM ceramics. Journal of dentistry. 2018;71:38-42.

7. Kurtulmus-Yilmaz S, Cengiz E, Ongun S, Karakaya I. The Effect of Surface Treatments on the Mechanical and Optical Behaviors of CAD/CAM Restorative Materials. Journal of prosthodontics : official journal of the American College of Prosthodontists. 2018.

8. Silva TM, Salvia AC, Carvalho RF, Pagani C, Rocha DM, Silva EG. Polishing for glass ceramics: which protocol? Journal of prosthodontic research. 2014;58(3):160-70.

9. Preis V, Grumser K, Schneider-Feyrer S, Behr M, Rosentritt M. Cycle-dependent in vitro wear performance of dental ceramics after clinical surface treatments. Journal of the mechanical behavior of biomedical materials. 2016;53:49-58.

10. Preis V, Behr M, Handel G, Schneider-Feyrer S, Hahnel S, Rosentritt M. Wear performance of dental ceramics after grinding and polishing treatments. Journal of the mechanical behavior of biomedical materials. 2012;10:13-22.

11. Sarac D, Sarac YS, Yuzbasioglu E, Bal S. The effects of porcelain polishing systems on the color and surface texture of feldspathic porcelain. The Journal of prosthetic dentistry. 2006;96(2):122-8.

12. Al-Wahadni AM, Martin DM. An in vitro investigation into the wear effects of glazed, unglazed and refinished dental porcelain on an opposing material. Journal of oral rehabilitation. 1999;26(6):538-46.

13. Bourke BM, Rock WP. Factors affecting the shear bond strength of orthodontic brackets to porcelain. British journal of orthodontics. 1999;26(4):285-90.

14. Obregon A, Goodkind RJ, Schwabacher WB. Effects of opaque and porcelain surface texture on the color of ceramometal restorations. The Journal of prosthetic dentistry. 1981;46(3):330-40.

15. Motro PF, Kursoglu P, Kazazoglu E. Effects of different surface treatments on stainability of 
ceramics. The Journal of prosthetic dentistry. 2012;108(4):231-7.

16. Pecho OE, Perez MM, Ghinea R, Della Bona A. Lightness, chroma and hue differences on visual shade matching. Dental materials : official publication of the Academy of Dental Materials. 2016;32(11):1362-73.

17. Barghi N. Color and glaze: effects of repeated firings. The Journal of prosthetic dentistry. 1982;47(4):393-5.

18. Douglas RD, Steinhauer TJ, Wee AG. Intraoral determination of the tolerance of dentists for perceptibility and acceptability of shade mismatch. The Journal of prosthetic dentistry. 2007;97(4):2008.

19. Samra AP, Pereira SK, Delgado LC, Borges CP. Color stability evaluation of aesthetic restorative materials. Brazilian oral research. 2008;22(3):20510.

20. Guler AU, Kurt S, Kulunk T. Effects of various finishing procedures on the staining of provisional restorative materials. The Journal of prosthetic dentistry. 2005;93(5):453-8.

21. Odioso LL, Gibb RD, Gerlach RW. Impact of demographic, behavioral, and dental care utilization parameters on tooth color and personal satisfaction. Compendium of continuing education in dentistry (Jamesburg, NJ : 1995) Supplement. 2000(29):S35-41; quiz S3.

22. Esquivel JF, Chai J, Wozniak WT. Color stability of low-fusing porcelains for titanium. The International journal of prosthodontics. 1995;8(5):479-85.

23. Jagger DC, Harrison A. An in vitro investigation into the wear effects of unglazed, glazed, and polished porcelain on human enamel. The Journal of prosthetic dentistry. 1994;72(3):320-3.

24. al-Wahadni A, Martin DM. Glazing and finishing dental porcelain: a literature review. Journal (Canadian Dental Association). 1998;64(8):580-3.
25. Kawai K, Urano M, Ebisu S. Effect of surface roughness of porcelain on adhesion of bacteria and their synthesizing glucans. The Journal of prosthetic dentistry. 2000;83(6):664-7.

26. Raimondo RL, Jr., Richardson JT, Wiedner B. Polished versus autoglazed dental porcelain. The Journal of prosthetic dentistry. 1990;64(5):553-7.

27. Fuzzi M, Zaccheroni Z, Vallania G. Scanning electron microscopy and profilometer evaluation of glazed and polished dental porcelain. The International journal of prosthodontics. 1996;9(5):452-8.

28. Patterson CJ, McLundie AC, Stirrups DR, Taylor WG. Refinishing of porcelain by using a refinishing kit. The Journal of prosthetic dentistry. $1991 ; 65(3): 383-8$.

29. Klausner LH, Cartwright CB, Charbeneau GT. Polished versus autoglazed porcelain surfaces. The Journal of prosthetic dentistry. 1982;47(2):157-62.

30. Goldstein GR, Barnhard BR, Penugonda B. Profilometer, SEM, and visual assessment of porcelain polishing methods. The Journal of prosthetic dentistry. 1991;65(5):627-34.

31. Okubo SR, Kanawati A, Richards MW, Childress S. Evaluation of visual and instrument shade matching. The Journal of prosthetic dentistry. 1998;80(6):642-8.

32. Lim HN, Yu B, Lee YK. Spectroradiometric and spectrophotometric translucency of ceramic materials. The Journal of prosthetic dentistry. 2010;104(4):239-46.

33. Atay A, Karayazgan B, Ozkan Y, Akyil MS. Effect of colored beverages on the color stability of feldspathic porcelain subjected to various surface treatments. Quintessence Int. 2009;40(7):e41-8.

34. Ayad NM. Susceptibility of restorative materials to staining by common beverages: an in vitro study. Eur J Esthet Dent. 2007;2(2):236-47. 